\title{
Numerical Simulation of Probe Measurements in a Non- equilibrium Plasma, Using a Detailed Model Electron Fluid
}

\author{
Jeremiah Boerner" and Iain D. Boyd ${ }^{\dagger}$ \\ Department of Aerospace Engineering, University of Michigan, Ann Arbor, MI, 48109
}

\begin{abstract}
This study investigates the axisymmetric plasma flow field near a Faraday probe. A hybrid fluid PIC computational code simulates heavy particles with a Particle In Cell (PIC) model, and electrons with a detailed model derived from conservation of mass, momentum, and energy. These simulations show significant departures from the planar Bohm sheath solution and from previous simulations using the Boltzmann model electron fluid. In the current work, the sheaths extend three to six Debye lengths from the probe depending on the properties of the ion distribution. This is significantly more compact than seen in the Bohm sheath solution and previous simulations, which show sheaths that extend twice as far from the probe. Simulated measurements of ion current at the probe surface do not change as much, only increasing by $1 \%$ over Bohm sheath solution values. Variation in the conductivity of the plasma is believed to be the main source of the discrepancies.
\end{abstract}

\section{Nomenclature}

$e \quad=$ electron charge

$\mathbf{E} \quad=$ electric field

j $\quad=$ electron current

$k_{B} \quad=$ Boltzmann constant

$L_{D} \quad=$ Debye length

$M \quad=$ Mach number with respect to Bohm velocity

$m_{i} \quad=$ ion mass

$n_{e} \quad=$ electron number density

$n_{i} \quad=$ ion number density

$P_{e} \quad=$ electron pressure

$T_{e} \quad=$ electron temperature

$T_{i} \quad=$ ion temperature

$v_{B} \quad=$ Bohm velocity

$\mathbf{v}_{\mathbf{e}} \quad=$ electron velocity

$\mathbf{v}_{\mathbf{i}} \quad=$ ion drift velocity

$x=$ sheath coordinate

$\varepsilon_{0} \quad=$ permittivity of free space

$\kappa=$ electron thermal conductivity

$\sigma \quad=$ electron conductivity

$\Phi \quad=$ local potential

$\Psi \quad=$ electron streamfunction

$v_{e} \quad=$ electron collision frequency

\section{Introduction}

GLECTRIC PROPULSION (EP) technology encompasses a wide variety of approaches to generate spacecraft - thrust through means other than chemical rockets. Devices can be grouped into one of three conceptual categories based on the mechanism for accelerating the propellant. ${ }^{1}$ Electrothermal devices such as resistojets and arcjets use electrical resistance to heat a propellant before expanding through a rocket nozzle. Electrostatic devices

\footnotetext{
*Graduate Student, Aerospace Engineering (jboerner@umich.edu).

${ }^{\dagger}$ Professor, Aerospace Engineering (iainboyd@ umich.edu).
} 
including ion thrusters and Hall thrusters apply external electric fields to accelerate ions. Electromagnetic devices such as magnetoplasmadynamic thrusters apply magnetic fields to accelerate ions through the $\mathbf{j} \times \mathbf{B}$ term of the Lorentz force.

In general, EP devices produce high exhaust velocities (and therefore large specific impulse) but low thrust. The large specific impulse can benefit long-duration missions by reducing the propellant mass requirement for a fixed mission profile, or by extending the operational lifetime for a fixed propellant mass. Alternatively, the low thrust increments attainable from EP systems can be useful in applications that require precision station keeping or maneuvering.

Continuing development of next-generation Hall thrusters and ion engines includes life tests, performance evaluation, and spacecraft integration. Erosion of components such as the discharge chamber walls in Hall thrusters or the ion optics in ion thrusters can lead to performance losses and eventually to failure of the EP system. Another important concern in the use of EP thrusters is the possible impingement of high-energy ions on the spacecraft, leading to material erosion or deposition on exposed surfaces. Experimental measurements of plasma properties in the exhaust plume are used to characterize the energy spectrum and particle flux, which determine sputter and deposition rates.

Several diagnostic instruments are used in these experiments, including Faraday probes, Langmuir probes, and retarding potential analyzers (RPA) ${ }^{2-4}$ These probes are immersed in the plasma and introduce both physical obstructions and electrostatic sheaths, which make it difficult to recover undisturbed plasma conditions from the measurements. Experimental tests performed in vacuum chambers must also contend with a background pressure due to pumping limitations. ${ }^{5}$ Neutral particles in the chamber lead to an overabundance of low energy charge exchange (CEX) ions, which are not expected in such high numbers during operation on orbit.

Previous numerical simulation studies suggest that the 1D Bohm sheath solution is a reliable predictor for instrument performance over a range of ambient plasma conditions. ${ }^{6-7}$ However, these studies are limited by use of the Boltzmann relation in the electron fluid model. The Boltzmann model requires electrons to be unmagnetized, isothermal, currentless, and collisionless. In most EP thruster plumes, the electrons are not isothermal, currentless or collisionless, and may be magnetized in the near-field of electrostatic or electromagnetic devices. Furthermore, these simulations are limited to ion collecting modes, since electron behavior is not evaluated correctly near positively biased probe surfaces.

This paper describes the use of a detailed model electron fluid in a hybrid fluid PIC code. First, the planar Bohm sheath solution is described as a baseline for the simulation results. Second, the computational code is described, along with the governing equations for the Boltzmann model and the detailed model. Next, the probe geometry and inflowing plasma conditions are outlined. New results using the detailed model are then presented and compared to the Bohm sheath solution and to previous Boltzmann model hybrid-PIC simulations. The impact of the detailed model is then evaluated by reviewing the simulated collected current measurement at the probe surface.

\section{Planar Bohm Sheath Solution}

The presence of a fixed potential surface in the plasma causes a sheath to form. The sheath structure consists of undisturbed neutral plasma far from the surface, an approximately neutral presheath region with small potential gradient, and a non-neutral sheath region with large potential gradient within a few Debye lengths of the surface. An analytic solution exists for the simple case of a collisionless neutral plasma flowing with uniform velocity toward an infinite surface held at a fixed potential.

For monoenergetic ions in a collisionless planar sheath, continuity and conservation of energy can be manipulated to express the local ion density $n_{i}(x)$ in terms of the local potential $\phi(x)$ and the ion density $n_{i s}$ and velocity $\boldsymbol{v}_{\text {is }}$ at the sheath edge.

$$
n_{i}(x)=n_{i s}\left(1-\frac{2 e}{m_{i} v_{i s}^{2}} \phi(x)\right)^{-\frac{1}{2}}
$$

The electrons are assumed to follow a Maxwellian distribution in a steady sheath solution. The local electron density $n_{e}(x)$ is then determined from the potential by the Boltzmann relation.

$$
n_{e}(x)=n_{i s} \exp \left(\frac{e \phi(x)}{k_{B} T_{e}}\right)
$$


Poisson's equation closes the set by relating the potential to the ion and electron number densities in the sheath.

$$
\frac{d^{2} \phi(x)}{d x^{2}}=-\frac{e}{\varepsilon_{0}}\left(n_{i}(x)-n_{e}(x)\right)
$$

A second-order differential equation for the potential is obtained when Eqs. (1)-(2) are combined into Eq. (3). If the electron temperature is used to normalize the potential, the Debye length $L_{D}$ and Bohm velocity $v_{B}$ appear as appropriate length and velocity scales, and the sole remaining parameter is the Mach number based on Bohm velocity $M .^{6}$ The coordinate system begins with $x=0$ at the sheath edge, where the boundary conditions are:

$$
\begin{gathered}
\phi(0)=0 \text { and } \frac{d \phi}{d x}(0) \approx 0 \\
n_{i}(0)=n_{e}(0)=n_{i s}
\end{gathered}
$$

This differential equation can be integrated once analytically, but requires numerical techniques to complete the second integration and find the potential. The ion and electron densities and velocities can then be determined from the local potential at any point throughout the sheath. The preceding analysis considers only electrons and monoenergetic single charge ions, but it is a straightforward exercise to generalize this result for multiple charge ions and more than one inflowing velocity.

\section{Computational Model Description}

Our computational model simulates 2D axisymmetric flow using a hybrid fluid Particle In Cell (PIC) method. Ions and neutrals are treated with a PIC model, ${ }^{8}$ while electrons are simulated with one of the fluid models described below. A direct simulation Monte Carlo (DSMC) routine ${ }^{9}$ is in place to handle collisions, although the plasma conditions in this study are very nearly collisionless. Particle weight (the number of real atoms represented by a simulated particle) is varied in steps from the centerline to the outer edge of the domain in order to limit the number of simulated particles and reduce the total computation time. Macroscopic properties are determined from weighted averages of the particles within a cell.

At the upstream edge, an inlet boundary generates the ion and neutral particles that enter the domain during a time step. The velocity of each particle is selected from a Maxwellian distribution using an acceptance/rejection method, with thermal temperature and drift velocity included as inputs. For simulations with two or more inflowing species, properties for each species are sampled from a separate distribution. Gradients of potential and electron streamfunction are specified along this edge. The outer radial edge also has an inlet boundary condition and introduces particles from the same distributions as the upstream inlet. Along this edge, the radial component of gradient is set to zero for all properties.

Ion particles undergo diffuse reflection and are converted to neutral particles at probe surfaces. Charged particle collisions with the collecting surface are recorded and weighted by the area of the impacted cell edge. Summing these area-weighted collisions over the collecting surface gives the simulated collected current. The collected ion and electron currents are averaged during the sampling process along with other macroscopic properties.

Two models for the electron fluid are considered here. The first is a Boltzmann model, where a series of assumptions about the electron fluid reduces the momentum equation to the Boltzmann relation. The second is a detailed model that retains electron continuity, momentum, and energy equations for the fluid. By taking the appropriate limits on the detailed model it is possible to recover a Boltzmann model form. This provides a useful comparison to validate the operation of the detailed model.

Both models begin from the electron conservation equations: ${ }^{10}$

Continuity: $\frac{\partial}{\partial t}\left(n_{e}\right)+\nabla \cdot\left(n_{e} \mathbf{v}_{\mathbf{e}}\right)=0$

Momentum: $\frac{\partial}{\partial t}\left(m_{e} n_{e} \mathbf{v}_{\mathbf{e}}\right)+m_{e} n_{e} \mathbf{v}_{\mathbf{e}} \cdot \nabla \mathbf{v}_{\mathbf{e}}=-e n_{e} \mathbf{E}-\nabla P_{e}+\frac{e n_{e} \mathbf{j}}{\sigma}$

Energy: $\quad \frac{\partial}{\partial t}\left(\frac{3}{2} n_{e} k_{B} T_{e}\right)+\frac{3}{2} n_{e} k_{B} \mathbf{v}_{\mathbf{e}} \cdot \nabla T_{e}=\mathbf{j} \cdot \mathbf{E}-P_{e} \nabla \cdot \mathbf{v}_{\mathbf{e}}+\nabla\left(\kappa \nabla T_{e}\right)-3 \frac{m_{e}}{m_{i}} n_{e} v_{e} k_{B}\left(T_{e}-T_{i}\right)$ 
The derivation for both models begins by assuming a steady state and neglecting inertial terms, and expressing the electron pressure $P_{e}$ in terms of density and temperature using the ideal gas law. The continuity equation can then be written as a Poisson equation by introducing a streamfunction $\Psi$ defined as $\nabla \Psi=n_{e} \mathbf{v}_{\mathrm{e}}$ so that continuity reduces to

$$
\nabla^{2} \boldsymbol{\Psi}=0
$$

At this point, several simplifying assumptions can be made about the electron fluid, eventually leading to the Boltzmann model. Alternatively, the governing equations can be manipulated mathematically to obtain the detailed model.

\section{A. Boltzmann Model Electron Fluid}

By assuming the electron fluid is isothermal, currentless, and unmagnetized, the energy equation is eliminated completely, and the momentum equation can be reduced to terms involving only the electron density and potential. This can then be integrated to yield the Boltzmann relation,

$$
\phi(x)-\phi^{*}=\frac{k_{B} T_{e}}{e} \ln \left(\frac{n_{e}(x)}{n^{*}}\right)
$$

with the starred quantities indicating a reference potential and density. Here $\phi^{*}=0 \mathrm{~V}$ at $n_{e}=n_{i s}$.

A relation to the ion density is required in order to close this set of equations. One approach is to assume quasi-neutrality, so that the ion density can replace the electron density and the potential can be found from Eq. 7. Another approach is to solve Poisson's equation for the potential, and use Eq. 7 to find the electron density. The second approach was used in our previous near-probe simulations, with an Alternating Direction Implicit (ADI) solver to find a self-consistent potential field from the electron and ion densities. Electrostatic fields are calculated from the gradient of the potential and then applied to the particles.

\section{B. Detailed Model Electron Fluid}

Following the derivation outlined by Boyd and Yim, ${ }^{11}$ the electron momentum equation can be rearranged to this generalized Ohm's law form.

$$
\mathbf{j}=\sigma\left[\frac{1}{e n_{e}} \nabla\left(n_{e} k_{B} T_{e}\right)-\nabla \phi\right]
$$

The divergence of this expression represents a steady state conservation of charge. That result can be manipulated to yield a Laplace equation for the potential, with coupling to the energy equation through the electron temperature.

$$
\nabla \cdot \mathbf{j}=0 \rightarrow \nabla^{2} \phi=\frac{k_{B}}{e n_{e} \sigma} \nabla \cdot\left[\sigma \nabla\left(n_{e} T_{e}\right)\right]-\frac{1}{\sigma} \nabla \cdot(\sigma \nabla \phi)
$$

The energy equation can also be rearranged into a Laplace equation for the electron temperature, which is coupled back to the potential.

$$
\nabla^{2} T_{e}=\frac{1}{\kappa}\left[\frac{3}{2} n_{e} k_{B} \mathbf{v}_{\mathbf{e}} \cdot \nabla T_{e}+\mathbf{j} \cdot \nabla \phi+n_{e} k_{B} T_{e} \nabla \cdot \mathbf{v}_{\mathbf{e}}-\nabla \kappa \nabla T_{e}+3 \frac{m_{e}}{m_{i}} n_{e} k_{B} v_{e}\left(T_{e}-T_{i}\right)\right]
$$

The electrical conductivity $\sigma$ and thermal conductivity $\kappa$ are evaluated from basic definitions. ${ }^{11}$ Eqs. 8 and 9 must be solved iteratively to produce consistent potential and temperature fields.

The Boltzmann relation can be recovered from this point by taking the limits corresponding to the assumptions made in the Boltzmann model. In the limit of very large conductivity (currentless) and constant temperature (isothermal), the second term of Eq. 8 becomes negligible, as does the gradient of conductivity in the first term. This leaves 


$$
\nabla^{2} \phi=\frac{k_{B} T_{e}}{e} \frac{\nabla^{2} n_{e}}{n_{e}}
$$

which can be integrated to give the Boltzmann relation.

A relation to the ion density is also required to complete this model. In this study we solve for a consistent electron density according to Poisson's equation using the ion density from the PIC module and $\nabla^{2} \Phi$ calculated from the potential found by solving Eq. 8. Due to the statistical scatter in the PIC model, a running average of the ion density is used in order to maintain a stable (and positive!) electron density. The electron density is especially sensitive to statistical fluctuations in ion density or potential when the ion density is low. An alternative approach to close this set of equations would be to solve Eq. 8 for the electron density and then solve Poisson's equation for the potential. This process is closer to the approach used in our previous Boltzmann model simulations.

Application of the detailed model in these cases requires an update of the existing computational code to double precision variables. Computation of the discretized Poisson equation at single precision can become numerically unstable due to the difference in magnitudes between the density gradients $\left(\sim 10^{18} /\right.$ cell $)$ and the potential gradients $\left(\sim 10^{5} /\right.$ cell $)$ across the small computational cells. The increased memory requirement of using double precision variables is not prohibitive on the desktop computer used to run the simulations. The total time required to run the simulations does not increase significantly.

The double precision code is validated by repeating several simulations originally performed using the single precision code. Operation of the detailed model is then tested by taking the limits described in Section III to obtain the Boltzmann relation. That is, the detailed model functions are modified to maintain constant electron temperature and a large, constant conductivity, so that the detailed model operates as a pseudo-Boltzmann model. The output from these validation simulations is consistent with previous Boltzmann model results to within a few percent.

\section{Inflow Conditions and Computational Grid}

Although the Bohm sheath solution only considers an isothermal, single species ion distribution, a realistic EP plume is much more complex. For experiments in a vacuum chamber, there is typically a high-velocity beam population and a lower-velocity charge exchange (CEX) population, with single and multiple charge ions and neutrals from both populations. In this study we proceed from the simple case of a single charge low-temperature beam population and incrementally approach a more realistic inflow plasma.

Undisturbed plasma properties are representative of the flow downstream of a low power Hall thruster. In particular, we choose values from $50 \mathrm{~cm}$ downstream and $75^{\circ}$ offaxis in the exhaust plume of a Busek Co. "BHT-200" $200 \mathrm{~W}$ xenon Hall thruster. This is a region of interest since experimental measurements suggest that the majority of the ion flux is due to CEX ions; that is, low-energy ions formed by collisions downstream of the thruster's acceleration region. The properties of the plasma components are recorded in Table 1.

Double charge ions are assumed to account

Table 1. Distribution properties and characteristic dimensions for the ion and neutral population components in a simulated BHT-200 plume.

\begin{tabular}{lccrccr}
\hline \hline Species & $\begin{array}{c}n_{i} \\
10^{14} \mathrm{~m}^{-3}\end{array}$ & \multicolumn{1}{c}{$\begin{array}{c}v_{i} \\
\mathrm{~m} / \mathrm{s}\end{array}$} & \multicolumn{1}{c}{$\begin{array}{c}T_{i} \\
\mathrm{~K}\end{array}$} & $\begin{array}{l}L_{D} \\
\mathrm{~mm}\end{array}$ & \multicolumn{1}{c}{$\begin{array}{l}v_{B} \\
\mathrm{~m} / \mathrm{s}\end{array}$} & $M$ \\
Beam & 0.4316 & 2,381 & 11,600 & 1.132 & 855.3 & 2.78 \\
CEX & 1.2951 & 1,026 & 300 & 0.653 & 855.3 & 1.20 \\
Double & 0.0959 & 1,365 & 300 & 2.399 & 855.3 & 1.60 \\
All ions & 1.9185 & 1,365 & - & 0.551 & 855.3 & 1.60 \\
Neutral & 320. & 855.3 & 300 & - & - & - \\
\hline
\end{tabular}

for $10 \%$ of the total freestream current flux, drawn from the beam and CEX ion densities in proportion to the freestream mole fractions. The velocity of the double charge ion distribution is set to the bulk charge velocity in order to maintain a constant current flux in each of the simulations. A more rigorous velocity calculation would account for the greater velocity developed by double charge ions formed in the thruster (double charge beam ions) and the greater range of velocities associated with single charge exchange and double charge exchange collisions.

The cylindrical Faraday probe geometry lends itself to a computational grid consisting of equally spaced rectangular cells. The computational domain is shown schematically in Fig. 1, with the probe front surface divided into collecting region and guard ring. Most experimental configurations apply a single potential on the entire probe with the goal of producing a uniform sheath over the collecting surface. We follow that convention in this study and set the probe potential at $-5 \mathrm{~V}$, although it is possible to set different biases for the collecting surface and the guard ring. Values for probe potential and electron streamfunction are assigned along the edges of the probe. 
The Bohm sheath solution for the beam ions provides an estimate of the required domain size, suggesting an upstream length of $1.1 \mathrm{~cm}\left(10 L_{D}\right)$ for these conditions. The appropriate radial extension for the probe geometry is not so neatly provided, and is set to one quarter-radius beyond the probe edge. Previous experience with the computational code suggests that the maximum cell spacing should be at least a factor of 12 smaller than the Debye length based on the total ion density. Rounding for conservative values, the cells are dimensioned at $4 \times 10^{-5} \mathrm{~m}$ on a side. The final geometry extends 390 cells $(1.560 \mathrm{~cm})$ along the probe axis and 390 cells $(1.560 \mathrm{~cm})$ radially, with 238 elements $(0.952 \mathrm{~cm})$ along the collecting surface and 80 elements $(0.320 \mathrm{~cm})$ along the guard ring. Altogether there are 112,350 cells outside of the probe body. Dimensions of the probe front face are selected to match the experimental instrument in Ref. 12.

The simulation time step is selected so that the fastest ions travel less than one cell length per iteration. For the beam populations, ions that enter at twice the thermal speed beyond the drift velocity arrive at the probe with a velocity of $6,220 \mathrm{~m} / \mathrm{s}$. Dividing the cell length by this speed and rounding down sets the time step at $5 \times 10^{-9} \mathrm{~s}$.

Each probe simulation is run for 10,000 iterations to reach a steady state, followed by 20,000 sampled iterations. At steady state the particle count ranges from 1.6 million particles for component populations to 2.6 million particles for a composite distribution with double charged ions and neutrals. A simulation can typically be completed in $25-$ 30 hours when run on a $3.8 \mathrm{GHz}$ Pentium 4 system.

\section{Results}

In the detailed model simulations, departures from the Boltzmann model behavior are expected to stem from variations in electron temperature, thermal conductivity, or electrical conductivity. Since the plasma conditions in this study are nearly collisionless, the electron temperature is not expected to change significantly. However, the thermal and electrical conductivity are proportional to the electron density, and will change accordingly.

Predictions from the Bohm sheath model are evaluated by comparing radial-average profiles of the simulated plasma properties. Plasma potential and densities are averaged over the first 100 cells $(4 \mathrm{~mm})$ from the centerline at each fixed axial position to yield radial-average profiles in the sheath. Previous simulations using the Boltzmann model $^{6-7}$ have been in very close agreement with the Bohm sheath solutions.

The simplest distribution considered in this paper is a low temperature ion beam population, with $1.1 \times 10^{14} \mathrm{~m}^{-3}$ ion density, $2,381 \mathrm{~m} / \mathrm{s}$ drift velocity, and $300 \mathrm{~K}$ temperature. The electron temperature in this case stays constant at the freestream value of $1 \mathrm{eV}$, neglecting round-off error in the computations. The simulated electrical conductivity in Fig. 2 shows a marked decrease of $13 \%$ within $3 \mathrm{~mm}\left(\sim 3 L_{D}\right)$ of the probe, corresponding to the region where electrons are repelled from the negatively biased probe. Taken as a whole, the detailed model predicts a sheath that only extends about $3 L_{D}$ from the probe, as opposed to $6 L_{D}$ for the Bohm sheath solution.

Simulated potential in the plasma is seen to remain nearly at the freestream value beyond this same distance in Figs. 3-4. This requires a much steeper potential gradient, or equivalently a thinner sheath, than anticipated from the Bohm sheath solution or previous Boltzmann model simulations.

Similar behavior is seen in the simulated electron density (Figs. 5-6) and simulated ion density (Figs. 7-8). One difference between the ion and electron behavior is observed along the side of the probe. Ions are attracted toward the probe, so a wake structure develops in the ion density as ions diffuse toward the probe and are eventually neutralized. The electrons develop a sheath structure with a uniform thickness similar to that upstream of the probe face. Previous Boltzmann model simulations have shown a more wake-like structure in the electron density.

Decreased electrical conductivity near the probe is believed to cause the increased potential gradient. The same total potential difference is applied, but the lower conductivity reduces the electron response to the electric field. The field cannot penetrate as far into the plasma as a result, causing the potential gradient to increase. The ion and electron densities then remain at the freestream values until closer to the probe surface.

Subsequent simulations add ion or neutral component populations, ultimately building up to the full population in Table 1. These intermediate simulations show the same general trends as the beam ion and full population cases. Since no other new features appear in the intermediate simulations, they are not discussed in detail here.

The full population introduces neutrals, causing a significant change in the electrical conductivity. The scatter in the simulated neutral density contours of Fig. 9 is due to the much higher neutral particle weight. Statistical fluctuations in the cell particle count are magnified in the density calculation, so the density is slow to converge. Electron-neutral collisions dominate the electrical conductivity in this case, leading to the conductivity contours shown in Fig. 10. High neutral density in the ram region upstream of the probe decreases conductivity throughout the domain. Near the probe surface, the conductivity decreases still more as the electron density falls off.

The simulated potential (Figs. 11-12) shows no sign of the more complicated ion distribution, and decreases monotonically toward the probe. The sheath reaches $\sim 6 L_{D}$ from the probe in this case, but remains at $3 \mathrm{~mm}$ since 
the Debye length is smaller for the higher ion density. Since the current flux in the beam ion case is selected to match the full population case, the potential profile is very similar for both cases.

Simulated electron density (Figs. 13-14) for the full population also behaves consistently with the beam ion case. The electron sheath extends as far as the potential sheath, and the same sheath structure is observed along the side of the probe. Contours of the total ion density are depicted in Fig. 15, with profiles of the component ion populations separated in Fig. 16. Each of the ion species shows a sheath structure that is independent of the other species. Since there are almost no collisions, each particle species interacts only with the collective potential field.

The simulated collected ion current at the probe surface is reported in Table 2 for the beam ion and full population cases. In both cases the collected current

Table 2. Simulated collected ion current, $\mu \mathrm{A}$, as calculated from the Bohm sheath theory, Boltzmann model simulations or detailed model simulations.

\begin{tabular}{rrrr}
\hline \hline & Bohm & Boltzmann & Detailed \\
\hline Beam ion & 11.95 & 12.00 & 12.08 \\
Full population & 11.94 & 12.03 & 12.10 \\
\hline \hline
\end{tabular}
found in the detailed model simulations is only $\sim 1 \%$ higher than expected from Bohm sheath solution or previous Boltzmann model simulations. This is not surprising since the freestream particle flux is unchanged and all the ions reach the surface. Although the potential gradient is steeper, the ions experience the field for a shorter time, arriving at the probe with approximately the same final velocity.

\section{Conclusion}

A hybrid fluid PIC code using a detailed fluid model was used to simulate axisymmetric plasma flow near a constant potential surface representing a Faraday probe. Significant departures from the Bohm sheath solution are observed in the simulated potential, electron density, and ion density in the sheath upstream of the probe. However, simulated measurements of ion current at the probe surface are not significantly affected.

The most notable departure observed in the detailed model results is that the sheath has only half the extent anticipated from the Bohm sheath solution. One aspect of probe design is to minimize edge effects in order to develop a uniform sheath over the collecting surface. This is typically accomplished by calculating a sheath thickness from the Bohm sheath model, and using that thickness to size the guard ring. There is an extra margin in that guard ring size if the "real" sheath indicated by the detailed model is thinner than the Bohm sheath.

There is also a significant difference between the electron and ion density behavior along the side of the probe. This motivates additional study of the effect of the outer radial edge placement and the length of the probe. Effects along the side of the probe are likely to impact measurements at the back surface, which is of interest for interpreting measurements from reversed Faraday probes.

The approach used here solves the electron momentum equation (Eq. 8) for the potential, which may not include enough information about the ion distribution. That is, the ion density only enters the calculation indirectly through the calculation of the electron density. In addition, this approach requires an initial potential field and an average ion density field to produce physically meaningful results. The alternative approach of solving the electron momentum equation for the electron density and then solving Poisson's equation for the potential might yield a more robust solution process.

\section{References}

1. Jahn, R. G., Choueiri, E. Y., "Electric propulsion," Encyclopedia of Physical Science and Technology, Vol. 5, 3rd ed., Academic Press, 2002, pp. 125-141.

2. Fife, J. M., Hargus, W. A., et al., "Spacecraft Interaction Test Results of the High Performance Hall System SPT-140," AIAA-2000-3521, 36th AIAA/ASME/SAE/ASEE Joint Propulsion Conference, July 2000.

3. Hofer, R. R., Hass, J. M., Gallimore, A. D., "Ion Voltage Diagnostics in the Far-field Plume of a High Specific Impulse Hall Thruster," AIAA-2003-4556, 39th AIAA/ASME/SAE/ASEE Joint Propulsion Conference, July 2003.

4. King, L. B., Gallimore, A. D., Maresse, C. M., "Transport-Property Measurements in the Plume of an SPT-100 Hall Thruster," Journal of Propulsion and Power, Vol. 14, No. 3, May-June 1998, pp. 327-335.

5. Walker, M. L. R., Gallimore, A. D., "Hot Flow Pressure Map of a Vacuum Facility as a Function of Flow Rate to Study Facility Effects," IEPC-2003-077, 28th International Electric Propulsion Conference, March 2003.

6. Boerner, J., Boyd, I. D., "Numerical Simulation of Probe Measurements in Non-equilibrium Plasma," AIAA2005-4790, 36th AIAA Plasmadynamics and Lasers Conference, June 2005. 
7. Boerner, J., Boyd, I. D., "Numerical Simulation of Faraday Probe Measurements in a Multi-Component Nonequilibrium Plasma," IEPC-2005-085, 29th International Electric Propulsion Conference, November 2005.

8. Birdsall, C. K. and Langdon, A. B., Plasma Physics Via Computer Simulation, Adam Hilger Press, 1991.

9. Bird, G. A., Molecular Gas Dynamics and the Direct Simulation of Gas Flows, No. 42 in Oxford Engineering Science Series, Oxford University Press, 1994.

10. Mitchner, M. and Kruger, C. H., Partially Ionized Gases, (Wiley, New York, 1973.)

11. Boyd, I. D. and Yim, J. T., "Modeling of the Near Field Plume of a Hall Thruster," Journal of Applied Physics, Vol. 95, 2004, pp. 4575-4584.

12. Ma, Tammy, "Quantification with Two-Dimensional Spatial Resolution of the Ion Flux Emitted from a $200 \mathrm{~W}$ Hall Effect Thruster," California Institute of Technology, Pasadena, California, 2004, (unpublished). 


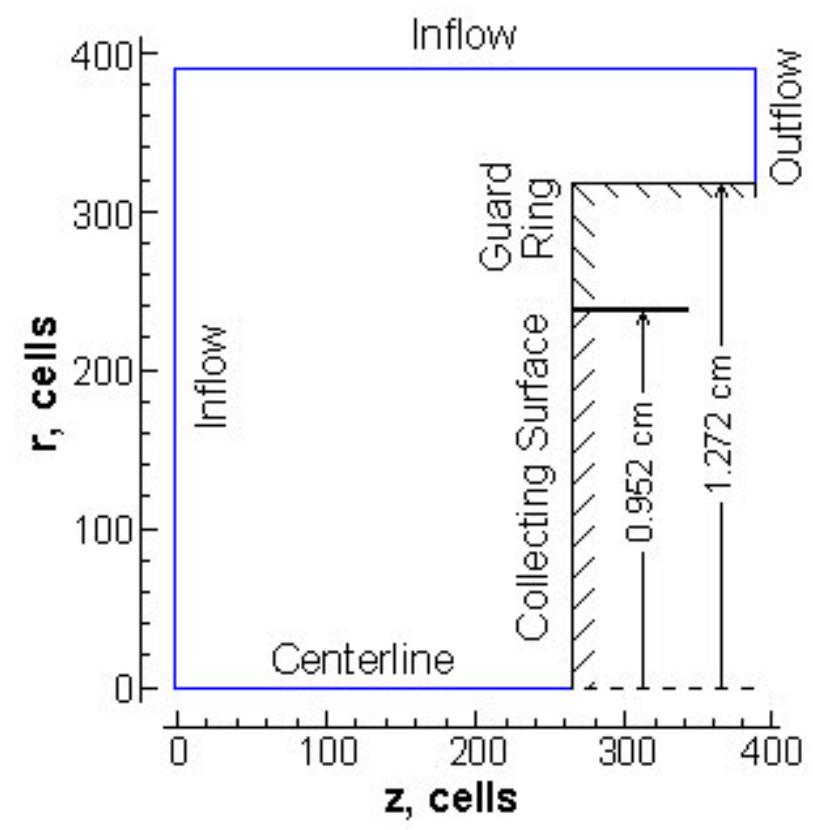

Figure 1. Computational domain, with probe surfaces at the right lower corner. Flow is from left to right.

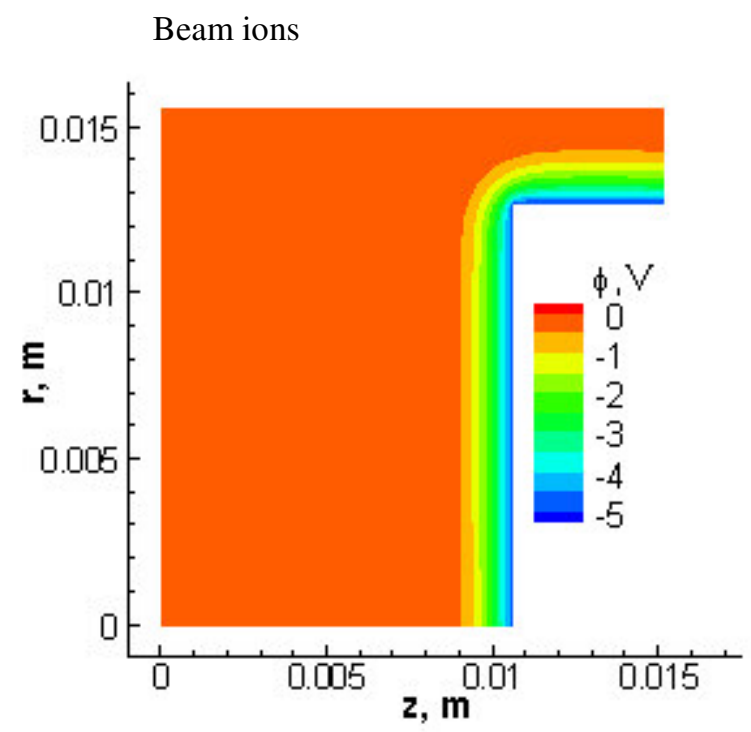

Figure 3. Contours of simulated potential remain near the freestream value until $3 L_{D}$ from the probe surface.

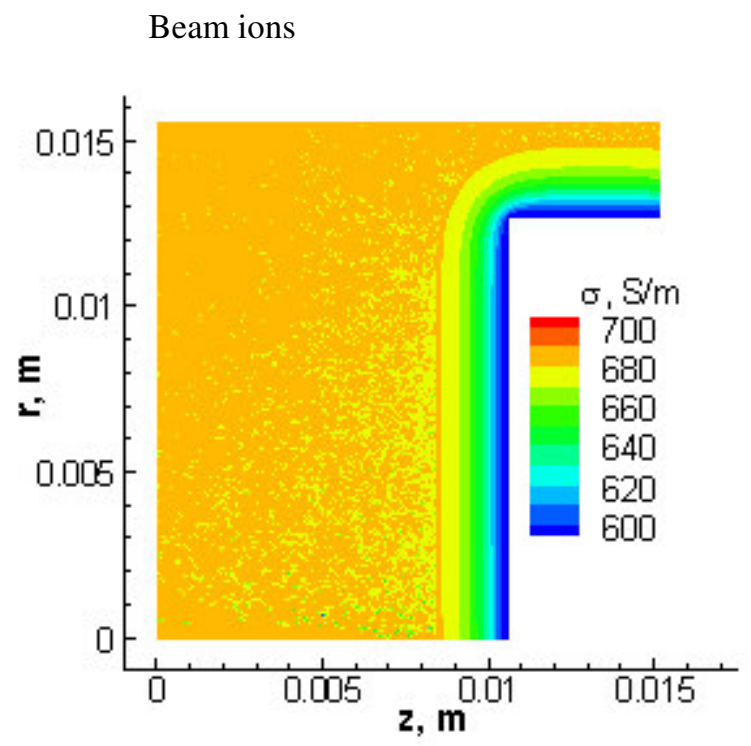

Figure 2. Contours of simulated electrical conductivity for the beam ion case, showing a decrease near the probe where electrons are repelled.

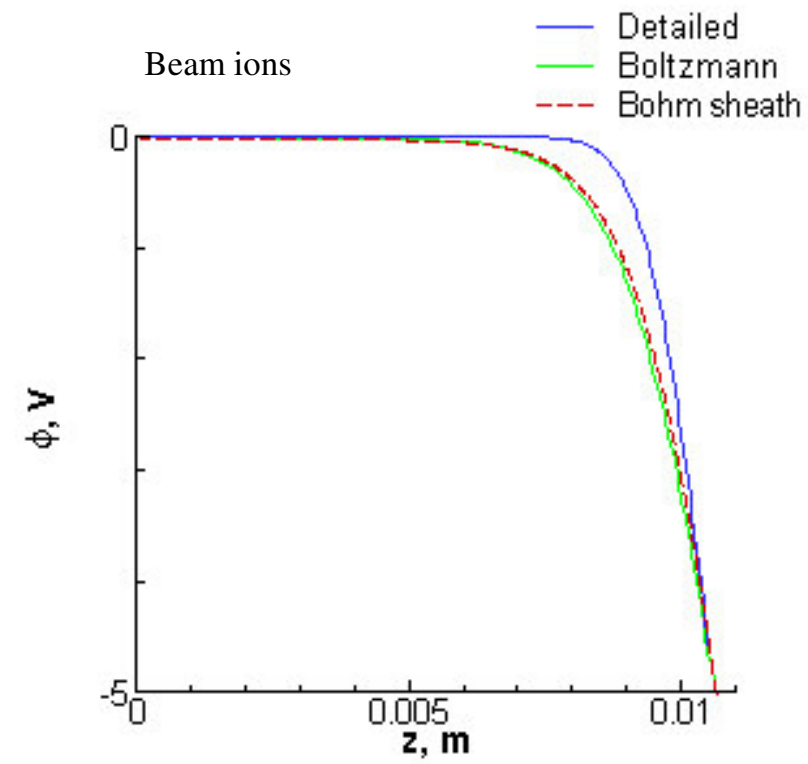

Figure 4. Radial average of simulated potential. Note that the detailed model results remain at the freestream condition much closer to the probe than in the Bohm sheath solution. 


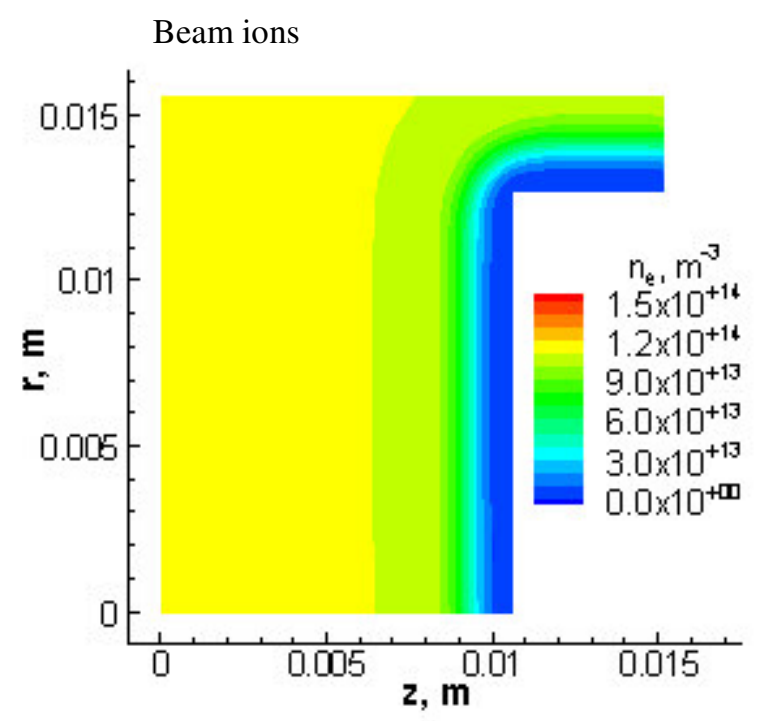

Figure 5. Contours of simulated electron density show a compact electron sheath along the side of the probe.

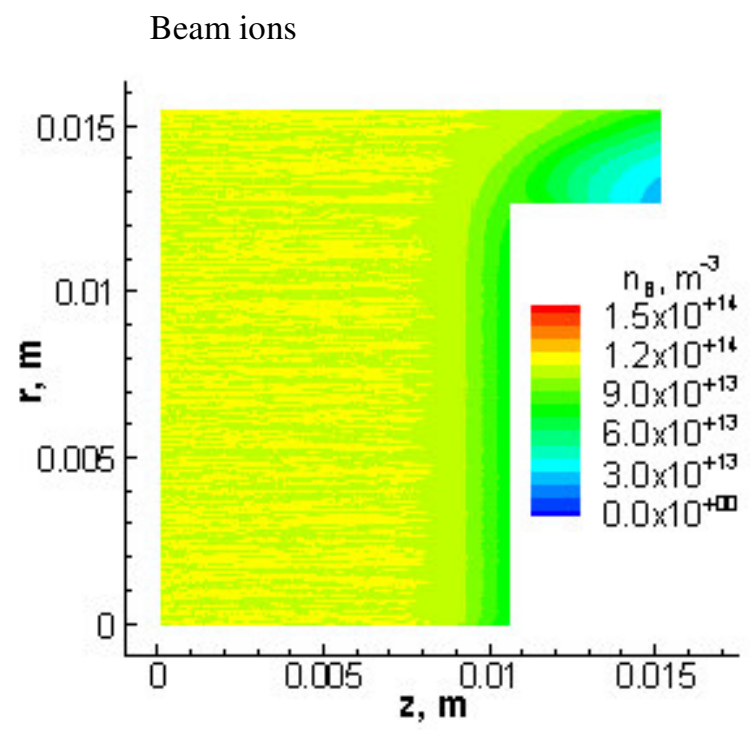

Figure 7. Contours of simulated ion density show a wake region along the side of the probe.

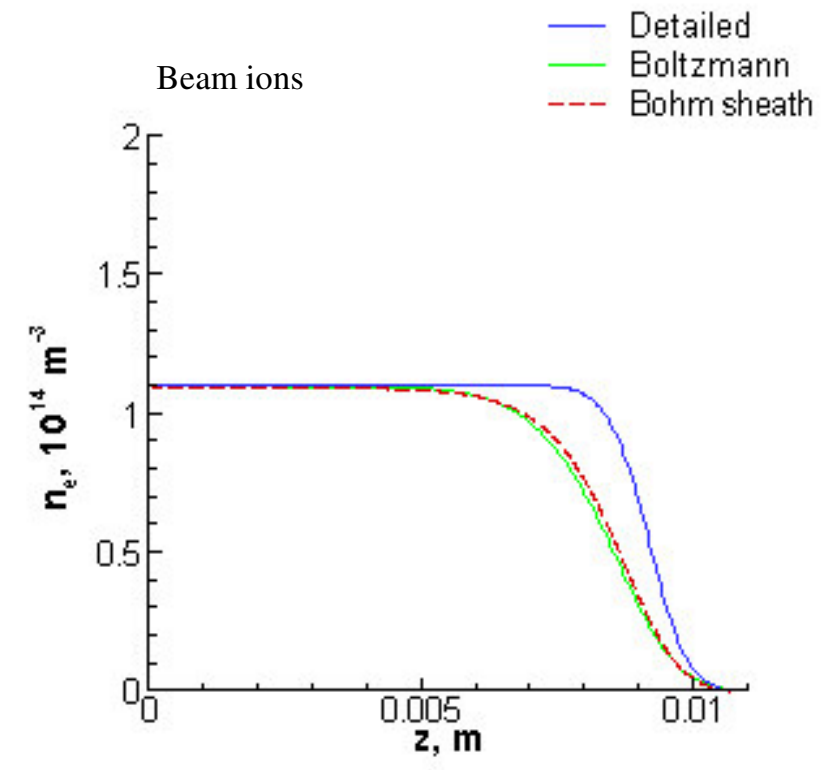

Figure 6. Radial average of simulated electron density.

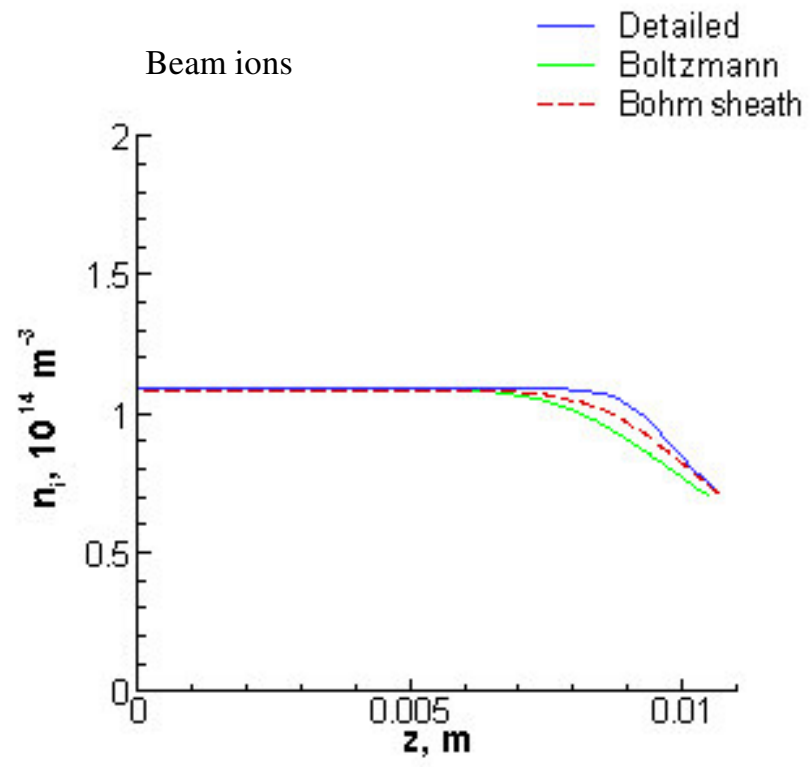

Figure 8. Radial average of simulated ion density. The Boltzmann model simulation gives a longer ion sheath due to non-zero temperature. 
Full population

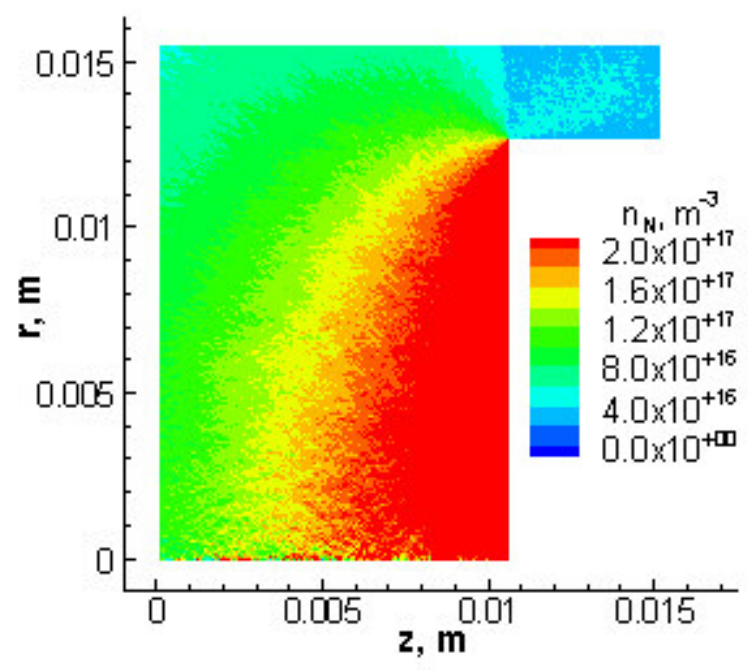

Figure 9. Contours of simulated neutral density for the full population from Table 1 . The scatter in these contours is due to large particle weight factor, leading to slow convergence of the average.

Full population

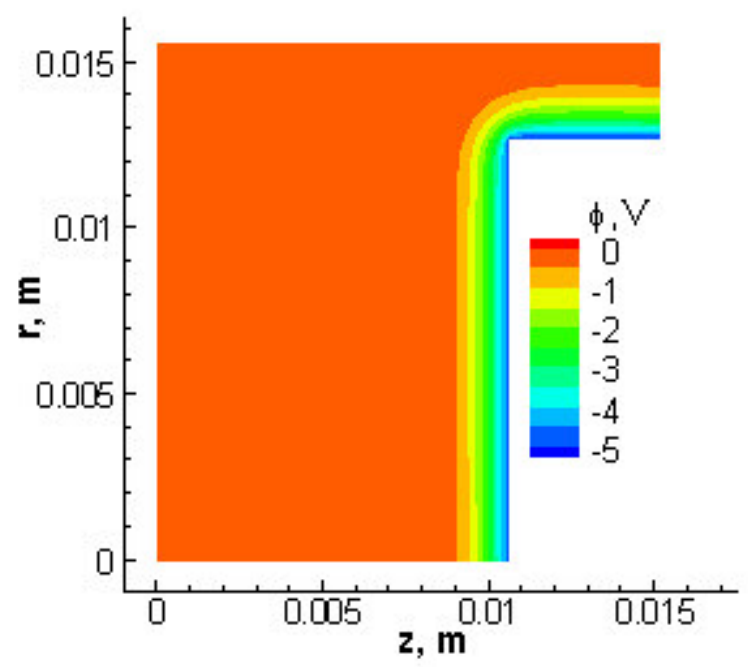

Figure 11. Contours of simulated potential remain at the freestream value until $6 L_{D}$ from the probe surface.
Full population

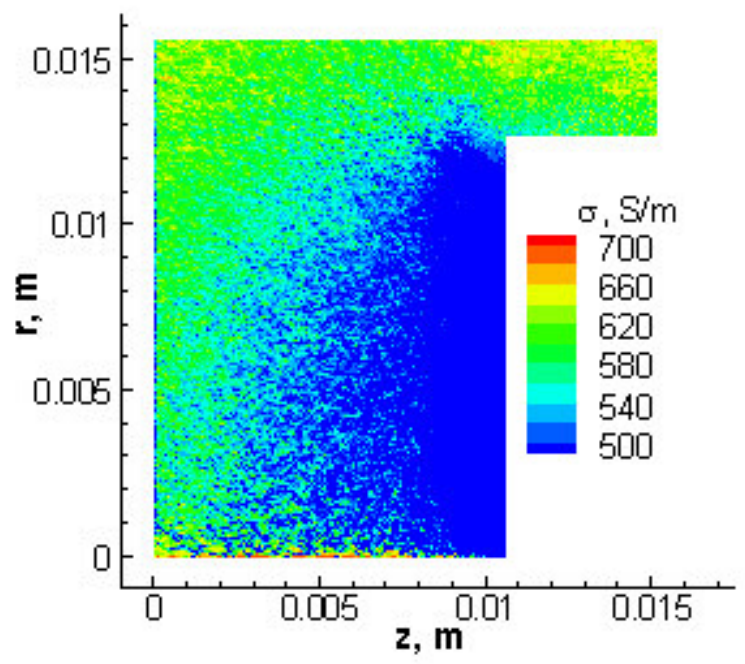

Figure 10. Contours of simulated electrical conductivity. Electron-neutral collisions drive the conductivity, leading to lower values throughout the domain.

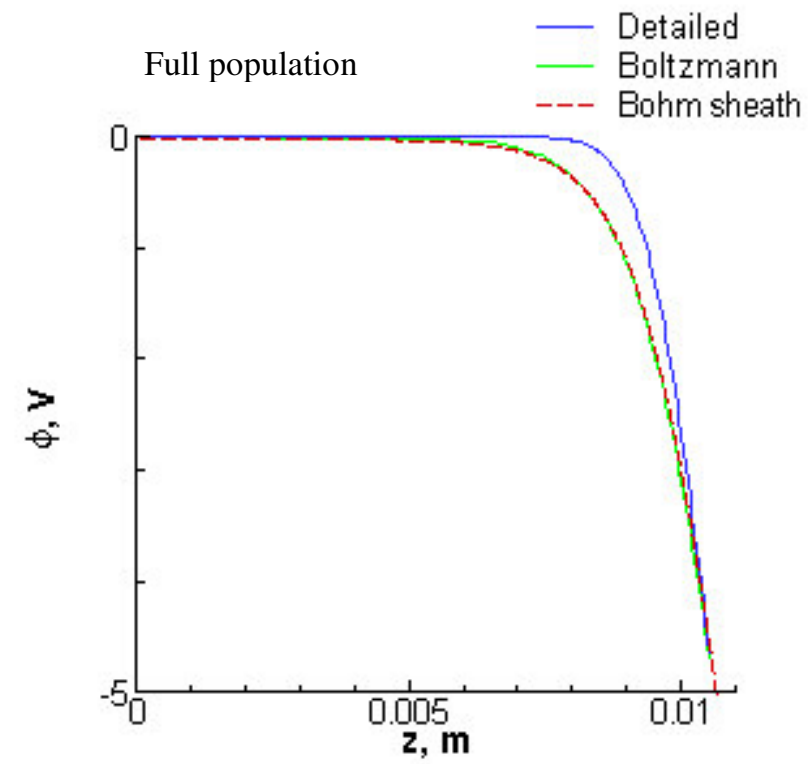

Figure 12. Radial average of simulated potential, again showing that the detailed model remains at the freestream conditions much closer to the probe than the Bohm sheath solution. 
Full population

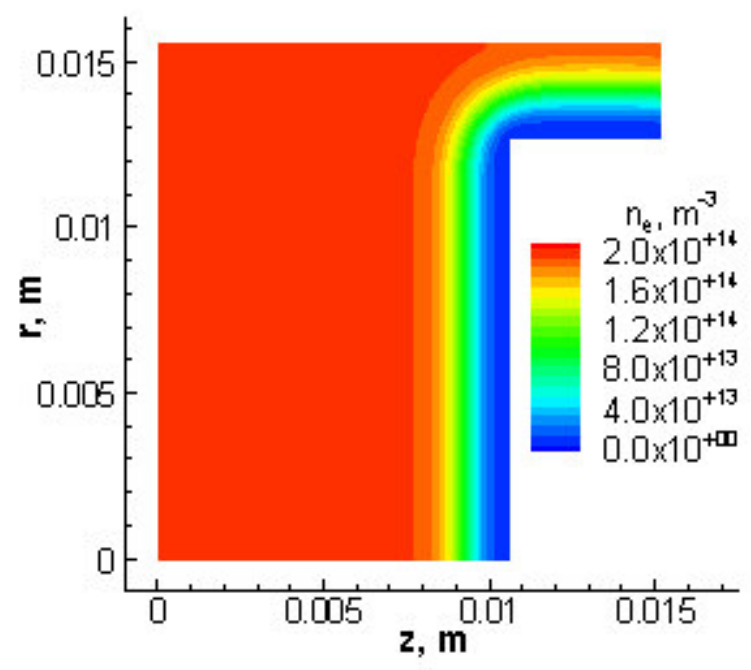

Figure 13. Contours of simulated electron density.

Full population

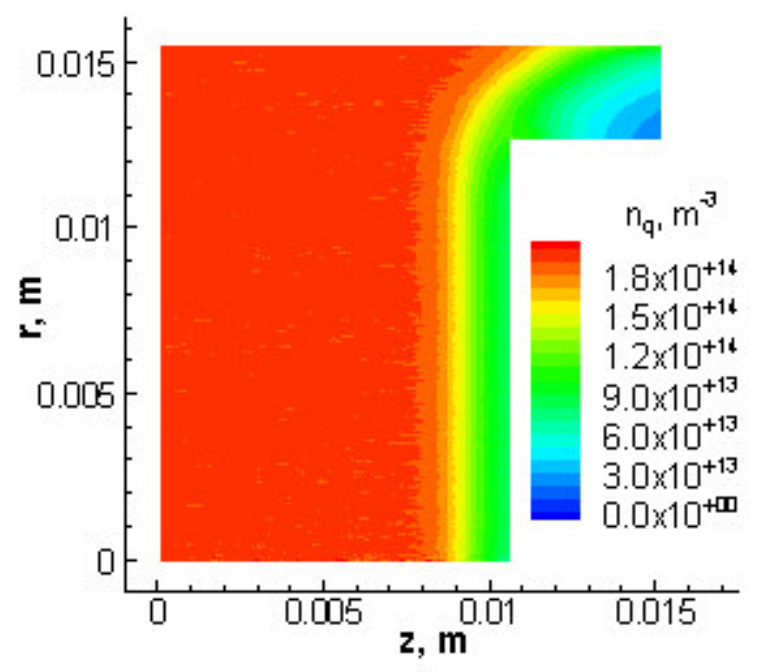

Figure 15. Contours of simulated ion density. Compare with Fig. 13, and note that the plasma is quasi-neutral over much of the domain.

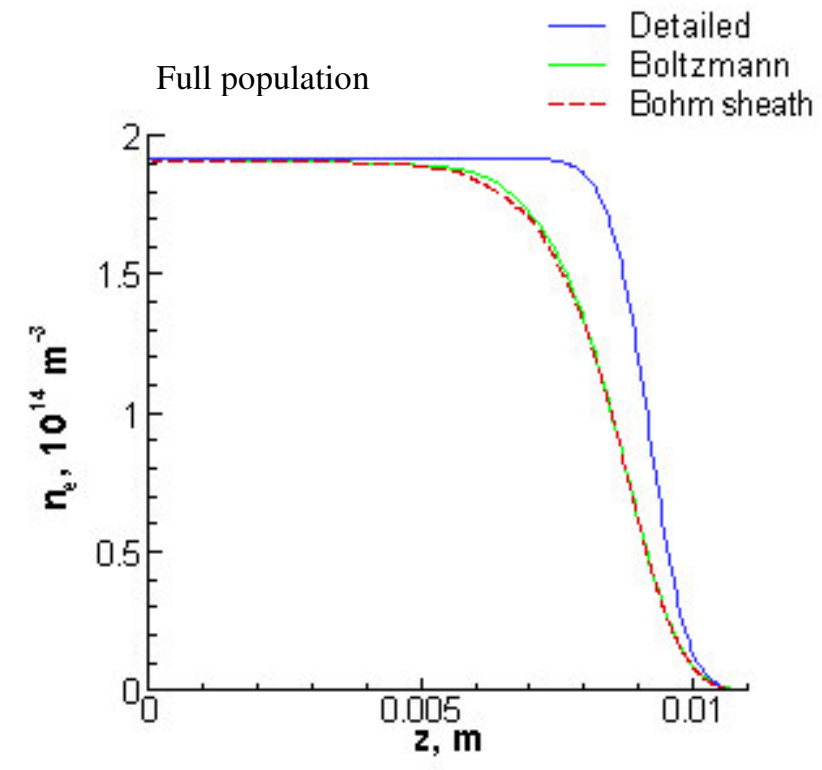

Figure 14. Radial average of simulated electron density.

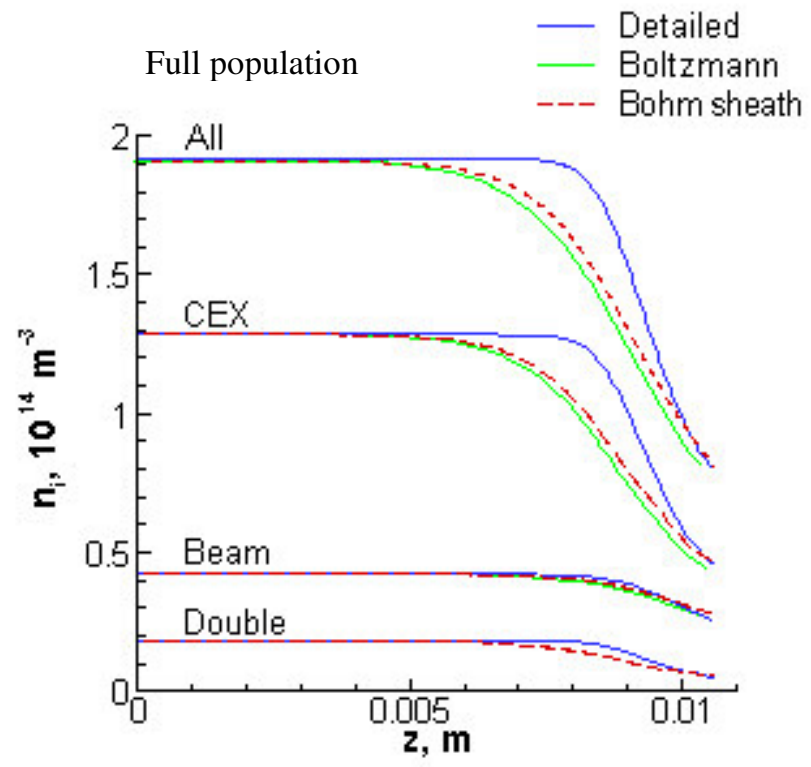

Figure 16. Radial average of simulated density for component ion species. Note that separate species have independent sheath lengths. 